\title{
Generalized wclosed sets in biweak structure spaces
}

\author{
H. M. Abu-Donia* and Rodyna A. Hosny
}

${ }^{*}$ Correspondence:

donia_1000@yahoo.com

Department of Mathematics,

Faculty of Science, Zagazig

University, Zagazig, Egypt

\begin{abstract}
As a generalization of the classes of gwclosed (resp. gwopen, sgwclosed) sets in a weak structure space $(X, W)$, the notions of $i j$-generalized $w$ closed (resp. ij-generalized wopen, ij-strongly generalized wclosed) sets in a biweak structure space $\left(X, W_{1}, w_{2}\right)$ are introduced. In terms of these concepts, new forms of continuous function between biweak spaces are constructed. Additionally, the concepts of ij-wnormal, ij-gwnormal, $i j-w T_{\frac{1}{2}}$, and $i j-W^{\sigma} T_{\frac{1}{2}}$ spaces are studied and several characterizations of them are acquired.
\end{abstract}

Keywords: Biweak structures, $i j-g w c l o s e d ~ s e t s, ~ i j-g\left(w, w^{\star}\right)$-continuous functions, ij-wnormal spaces

AMS classification: $54 \mathrm{~A} 05-54 \mathrm{C} 08-54 \mathrm{C} 10-54 \mathrm{C} 20$

\section{Introduction}

In recent years, many researchers studied bitopological, bigeneralized, biminimal, and biweak spaces due to the richness of their structure and potential for doing a generous area for the generalization of topological results in bitopological environment. The concept of a bitopological space was built by Kelly [1], and thereafter, an abundant number of manuscripts was done to generalize the topological notions to bitopological setting. Fukutake [2] presented the concept of generalized closed sets and in bitopological spaces. The notion has been studied extensively in recent years by many topologists. Csaszar and Makai Jr. proposed the concept of bigeneralized topology [3]. In 2010, Boonpok [4, 5] provided the concept of bigeneralized topological spaces and biminimal structure spaces, respectively. Csaszar [6] defined the concept of weak structure which is weaker than a supra topology, a generalized topology, and a minimal structure and then offered various properties of it. Ekici [7] have investigated further properties and the main rules of the weak structure space. In order to extend many of the important properties of $w$ closed sets to a larger family, Zahran et al. [8] characterized the concepts of generalized closed and generalized open sets in weak structures and achieved a number of properties of these concepts. As a generalization of bitopological spaces, bigeneralized topological spaces, and biminimal structure spaces, Puiwong et al. [9] in 2017 defined a new space, which is known as biweak structure. The concept of biweak structure can substitute in

(c) The Author(s). 2020 Open Access This article is licensed under a Creative Commons Attribution 4.0 International License, which permits use, sharing, adaptation, distribution and reproduction in any medium or format, as long as you give appropriate credit to the original author(s) and the source, provide a link to the Creative Commons licence, and indicate if changes were made. The images or other third party material in this article are included in the article's Creative Commons licence, unless indicated otherwise in a credit line to the material. If material is not included in the article's Creative Commons licence and your intended use is not permitted by statutory regulation or exceeds the permitted use, you will need to obtain permission directly from the copyright holder. To view a copy of this licence, visit http://creativecommons.org/licenses/by/4.0/. 
many situations, biminimal structures and bigeneralized topology. A new space consists of a nonempty set $X$ equipped with two arbitrary weak structures $w_{1}, w_{2}$ on $X$. A triple $\left(X, w_{1}, w_{2}\right)$ is called a biweak structure space (in short, biwss).

The interior (resp. closure) of a subset $A$ with respect to $w_{j}$ are denoted by $i n t_{w_{j}}(A)$ (resp. $\left.c l_{w_{j}}(A)\right)$, for $(j=1,2)$. A subset $A$ of a biwss $\left(X, w_{1}, w_{2}\right)$ is called $i j-w c l o s e d$ if $c l_{w_{i}}\left(c l_{w_{j}}(A)\right)=A$, where $i, j=1$ or 2 and $i \neq j$. The complement of an $i j$-wclosed set is called ij-wopen.

The concepts of generalized closed sets in weak structures [8] and biweak structure spaces [9] motivated us to define a new class of sets which is called generalized $w$ closed sets in a biweak structure space which are found to be effective in the study of digital topology. The purpose of this article is introducing the notions of $i j$-generalized $w c l o s e d$ (written henceforth as $i j$-gwclosed), $i j$-generalized wopen (written henceforth as $i j$-gwopen), and $i j$-strongly generalized wclosed ( $i j-\sigma g w c l o s e d$, for short) sets in a biwss $\left(X, w_{1}, w_{2}\right)$ as a generalization of the concept of $g w c l o s e d, g w o p e n$, and $s g w c l o s e d ~ s e t s$, respectively, in a weak structure space $(X, w)$ which presented in [8] and determining some of their behaviors. In terms of $i j-g w$ closed and $i j$-gwopen sets, new forms of continuous function between biweak spaces are constructed. Additionally, we try to extend the concepts of separation axioms on weak structures [8] to biwss and study some of their features. Some considerable results in articles $[2,8,10]$ can be treated as particular cases of our outcomes.

\section{Preliminaries}

To prepare this article as self-contained as possible, we recollect the next definitions and results which are due to various references $[8,9,11]$.

Definition 1 [8] Let $w$ be a weak structure on X. Then,

(1) A subset $A$ is called generalized wclosed (gwclosed, for short) if $c l_{w}(A) \subseteq U$, whenever $A \subseteq U$ and $U$ is wopen.

(2) The complement of a generalized wclosed set is called generalized wopen (gwopen, for short), i.e, a subset $A$ is gwopen if and only if $\operatorname{int}_{w}(A) \supseteq F$, whenever $A \supseteq F$ and $F$ is wclosed.

The family of all gwclosed (resp. gwopen) sets in a weak structure $X$ will be denoted by GWC $(X)($ resp. GWO $(X))$.

Definition 2 [11] Let $w$ and $w^{\star}$ be weak structures on $X$ and $Y$, respectively. A function $f:(X, w) \longrightarrow\left(Y, w^{\star}\right)$ is called $\left(w, w^{\star}\right)$-continuous if for $x \in X$ and $w^{\star}$ open set $V$ containing $f(x)$, there is wopen set $U$ containing $x$ s.t. $f(U) \subseteq V$.

Theorem 1 [11] Let $w$ and $w^{\star}$ be weak structures on $X$ and $Y$, respectively. For a function $f:(X, w) \longrightarrow\left(Y, w^{\star}\right)$, the following statements are equivalent:

(1) $f$ is $\left(w, w^{\star}\right)$-continuous,

(2) $f^{-1}(B)=\operatorname{int}_{w}\left(f^{-1}(B)\right)$, for every $w^{\star}$ open set $B$ in $Y$,

(3) $f\left(c l_{w}(A)\right) \subseteq c l_{w^{\star}}(f(A))$, for every set $A$ in $X$,

(4) $c l_{w}\left(f^{-1}(B)\right) \subseteq\left(f^{-1}\left(c l_{w^{\star}}(B)\right)\right.$, for every set $B$ in $Y$,

(5) $f^{-1}\left(\operatorname{int}_{w^{\star}}(B)\right) \subseteq i n t_{w}\left(f^{-1}(B)\right)$, for every set $B$ in $Y$, 
(6) $\quad c l_{w}\left(f^{-1}(F)\right)=f^{-1}(F)$, for every $w^{\star}$ closed set $F$ in $Y$.

Theorem 2 [9] Let $\left(X, w_{1}, w_{2}\right)$ be a biwss and $A$ be a subset of $X$. Then, the following are equivalent:

(1) $A$ is ij-wclosed,

(2) $A=c l_{w_{i}}(A)$ and $A=c l_{w_{j}}(A)$,

(3) $A=c l_{w_{j}}\left(c l_{w_{i}}(A)\right)$, where $i, j=1$ or 2 and $i \neq j$.

Proposition 1 [9] Let $\left(X, w_{1}, w_{2}\right)$ be a biwss and $A \subseteq X$. Then, $A$ is a ij-wclosed set, if $A$ is both $w_{i}$ closed and $w_{j}$ closed, where $i, j=1$ or 2 and $i \neq j$.

Proposition 2 [9] Let $\left(X, w_{1}, w_{2}\right)$ be a biwss. If $A_{\alpha}$ is ij-wclosed for all $\alpha \in \Lambda \neq \emptyset$, then $\cap_{\alpha \in \Lambda} A_{\alpha}$ is ij-wclosed and the union of two ij-wclosed sets is not a $i j$-wclosed set, where $i, j=$ 1 or 2 and $i \neq j$.

In the rest of this article $i, j$ will stand for fixed integers in the set $\{1,2\}$ and $i \neq j$.

\section{On ij-gwclosed sets}

In this part, a new family of sets called $i j$-generalized wclosed (briefly, $i j$-gwclosed) is presented and its properties are investigated.

Definition $3 A$ subset $A$ of a biwss $\left(X, w_{1}, w_{2}\right)$ is called ij-generalized wclosed (ijgwclosed, for short) if $c l_{w_{j}}(A) \subseteq U$, whenever $A \subseteq U$ and $U$ is $w_{i}$ open. The complement of ij-gwclosed set is called ij-gwopen.

The family of all ij-gwclosed (resp. ij-gwopen) sets in a biwss $\left(X, w_{1}, w_{2}\right)$ will be denoted by $i j-G W C(X)$ (resp. $i j-G W O(X))$.

Remark 1 If $A \in i j-G W C(X) \cap j i-G W C(X)$, then a subset $A$ of a biwss $\left(X, w_{1}, w_{2}\right)$ is called pairwise gwclosed and its complement is pairwise gwopen.

Example 1 Let $X=\{1,2,3\}, w_{1}=\{\emptyset,\{1\},\{1,2\}\}$, and $w_{2}=\{\emptyset,\{3\}\}$. A set $\{3\}$ is pairwise gwclosed.

Certainly, the next theorems are obtained:

Theorem 3 A subset $A$ of a biwss $\left(X, w_{1}, w_{2}\right)$ is ij-gwopen iff int $w_{j}(A) \supseteq F$, whenever $A \supseteq F$ and $F$ is $w_{i}$ closed.

Theorem 4 If $A$ is an ij-gwclosed and $w_{i}$ open set in $\left(X, w_{1}, w_{2}\right)$, then $A=c l_{w_{j}}(A)$.

Theorem 5 Every $w_{j}$ closed set in a biwss $\left(X, w_{1}, w_{2}\right)$ is ij-gwclosed.

Proof Let $A$ be a $w_{j}$ closed set and $U$ be a $w_{i}$ open set in $X$ s.t. $A \subseteq U$. Then, $c l_{w_{j}}(A)=A$. It implies that $A \in i j-G W C(X)$.

Corollary 1 If $A$ is a $w_{j}$ open set in a biwss (X, $\left.w_{1}, w_{2}\right)$, then $A \in i j-G W O(X)$. 
Remark 2 By the following example, we have a tendency to show that the converse of Theorem 5 is not always true.

Example 2 In Example 1, a set $\{2\}$ is 12-gwclosed and not $w_{2}$ closed.

Proposition 3 Let $\left(X, w_{1}, w_{2}\right)$ be a biwss. Then,

(1) If $X \in w_{j}$ and each $w_{i}$ open set is $w_{j}$ closed, then, $A \in i j-G W C(X)$, for each $A \subset X$.

(2) $A \in i j-G W C(X)$, for each $A \subset X$ iff $c l_{w_{j}} U=U$ for each $w_{i}$ open set $U$.

Proof We prove only (2) and the rest of the proof is simple. Suppose that $A \in i j$ $G W C(X)$, for each $A \subset X$. Then, every $w_{i}$ open set $U, A \in i j-G W C(X)$. If $U \subseteq U$, hence $c l_{w_{j}}(U) \subseteq U$. Thus, $c l_{w_{j}}(U)=U$, for each $w_{i}$ open set $U$. Conversely, suppose that $A \subseteq U$ and $U$ be a $w_{i}$ open set. Then, $c l_{w_{j}}(A) \subseteq c l_{w_{j}}(U)$. From assumption, $c l_{w_{j}}(A) \subseteq U$ and so $A \in i j-G W C(X)$.

Remark 3 In the biwss $\left(X, w_{1}, w_{2}\right)$, the converse of the Proposition 3(1) need not be true in general as shown by the next example.

Example 3 Let $X=\{1,2,3\}, w_{1}=\{\emptyset,\{2\},\{1,3\}\}$, and $w_{2}=\{\emptyset, X,\{1\},\{2\},\{3\},\{1,2\},\{2,3\}\}$. One may notice that every subset of $X$ is 12-gwclosed, but $A=\{2\}$ is a $w_{1}$ open set in $X$ and it is not $w_{2}$ closed.

Remark 4 In general, 21-GWC $(X) \neq 12-G W C(X)$ as in Example 3.

Proposition 4 Let $\left(X, w_{1}, w_{2}\right)$ be a biwss. If $w_{1} \subseteq w_{2}$, then 21-GWC $(X) \subseteq 12-G W C(X)$.

Proof Straightforward.

The converse of the Proposition 4 is not true as seen from the next example.

Example 4 In Example 3, then 21-GWC $(X) \subseteq 12-G W C(X)$, but $w_{1} \nsubseteq w_{2}$.

Now, one can conclude attitudes relative to the union as well as the intersection of two $i j$-gwclosed sets in a biwss $\left(X, w_{1}, w_{2}\right)$.

Example 5 Let $X=\{1,2,3,4\}, w_{1}=\{\emptyset,\{3\},\{1,3\},\{1,3,4\},\{1,2,4\}\}$ and $w_{2}=\{\emptyset,\{2\},\{3\}$, $\{2,3,4\}\}$. Let us consider $A=\{2\}$ and $B=\{3\}$. Note that $A$ and $B$ are 21-gwclosed sets but its union is not 21-gwclosed.

Example 6 Let $X=\{1,2,3\}, w_{1}=\{\emptyset,\{1\},\{3\}\}$ and $w_{2}=\{\emptyset,\{1\}\}$. Consider two 21-gwclosed sets $A=\{1,2\}$ and $B=\{1,3\}$, then $A \cap B=\{1\}$ is not 21-gwclosed.

Theorem 6 Let $\left(X, w_{1}, w_{2}\right)$ be a biwss and $c_{w_{j}}(\emptyset)=\emptyset$. Then, the family of all ij-gwclosed sets is a biminimal structure in $X$.

Proof Obvious.

Theorem 7 Suppose $X \in w_{i}$. Then, $\{x\}$ is $w_{i}$ closed or $X \backslash\{x\} \in i j-G W C(X)$, for each $x \in X$. 
Proof Suppose that the singleton $\{x\}$ is not $w_{i}$ closed for some $x \in X$. Then, $X \backslash\{x\}$ is not $w_{i}$ open. Since $X$ is $w_{i}$ open set and $X \backslash\{x\} \subseteq X$. Hence, $X \backslash\{x\} \in i j-G W C(X)$.

Theorem 8 If $A \in i j-G W C(X)$, then $c_{w_{j}}(A) \backslash A$ contains no nonempty $w_{i} c l o s e d$.

Proof For an $i j$-gwclosed set $A$, let $S$ be a nonempty $w_{i}$ closed set s.t. $S \subseteq c l_{w_{j}}(A) \backslash A$. Then, $S \subseteq c l_{w_{j}}(A)$ and $S \subseteq X \backslash A$. Since $X \backslash S$ is $w_{i}$ open and $A$ is $i j$-gwclosed, then $c l_{w_{j}}(A) \subseteq X \backslash S$ or $S \subseteq X \backslash c l_{w_{j}}(A)$. Thus, $S=\emptyset$. Therefore, $c l_{w_{j}}(A) \backslash A$ does not contain nonempty $w_{i}$ closed.

Remark 5 In general, the converse of Theorem 8 is not true as shown in the next example.

Example 7 In Example 6, if $A=\{1\}$, then $c_{w_{1}}(A) \backslash A=\{2\}$. So we know that there is no any nonempty $w_{2}$ closed contained in $c_{w_{1}}(A) \backslash A$. But $A \notin 21-G W C(X)$.

It thus follows from Theorem 8 that

Corollary 2 If $A \in i j-G W C(X)$ and $c l_{w_{j}}(A) \backslash A$ is a $w_{i}$ closed set, then $c l_{w_{j}}(A)=A$.

Remark 6 If $A$ is an ij-gwclosed set in a biwss $\left(X, w_{1}, w_{2}\right)$ and $c l_{w_{j}}(A)=A$, then $c l_{w_{j}}(A) \backslash A$ need not to be $w_{i}$ closed as shown by the following example.

Example 8 Let $X=\{1,2,3\}, w_{1}=\{\emptyset,\{2\}\}$, and $w_{2}=\{\emptyset,\{1\},\{3\},\{1,2\}\}$. If $A=\{2\}$, one may notice that $c_{w_{2}}(A)=A$ and hence $c_{w_{2}}(A) \backslash A=\emptyset$, which is not $w_{1}$ closed.

Theorem 9 If $A \in i j-G W C(X)$, then $c_{w_{j}}(A) \backslash A \in i j-G W O(X)$.

Proof Let $A \in i j-G W C(X)$ and $F$ be a $w_{i}$ closed set s.t. $F \subseteq c l_{w_{j}}(A) \backslash A$. Then, by Theorem 8, we have $F=\emptyset$ and hence $F \subseteq i n t_{w_{j}}\left(c l_{w_{j}}(A) \backslash A\right)$. So by Theorem 3, we have $c l_{w_{j}}(A) \backslash A \in i j$ $G W O(X)$.

Remark 7 The converse of the Theorem 9 need not to be true in general as shown by the following example.

Example 9 In Example 6. If $A=\{1\}$, one may notice that $c l_{w_{1}}(A) \backslash A \in 21-G W O(X)$, but $A \notin 21-G W C(X)$.

Theorem 10 If $A \in i j-G W C(X)$ and $A \subseteq B \subseteq c l_{w_{j}}(A)$, then $B \in i j-G W C(X)$.

Proof Let $U$ be any $w_{i}$ open set s.t. $B \subseteq U$. Since $A \subseteq B$ and $A \in i j-G W C(X)$, then $c l_{w_{j}}(A) \subseteq U$. Since $B \subseteq c l_{w_{j}}(A)$, then we have $c l_{w_{j}}(B) \subseteq c l_{w_{j}} c l_{w_{j}}(A)=c l_{w_{j}}(A) \subseteq U$. Consequently $B \in i j-G W C(X)$.

Corollary 3 Let $\left(X, w_{1}, w_{2}\right)$ be a biwss. Then,

(1) If $A \in i j-G W O(X)$ and int $w_{j}(A) \subseteq B \subseteq A$, then, $B \in i j-G W O(X)$.

(2) $c l_{w_{j}}(A) \in i j-G W C(X)$ if $A \in i j-G W C(X)$.

(3) $\operatorname{int}_{w_{j}}(A) \in i j-G W O(X)$ if $A \in i j-G W O(X)$.

In view of Theorems 8 and 10 , the next theorem is valid. 
Theorem 11 Let $A$ be an ij-gwclosed set with $A \subseteq B \subseteq c l_{w_{j}}(A)$, then, $c l_{w_{j}}(B) \backslash B$ does not contain nonempty $w_{i}$ closed.

Theorem 12 If $A$ is an ij-gwopen set in $X$, then $U=X$ whenever $U$ is $w_{i}$ open and $\operatorname{int}_{w_{j}}(A) \cup(X \backslash A) \subseteq U$.

Proof Let $U$ be a $w_{i}$ open set in $X$ and $i n t_{w_{j}}(A) \cup(X \backslash A) \subseteq U$ for any $i j-g$ wopen set $A$. Then, $X \backslash U \subseteq\left(X-\operatorname{int}_{w_{j}}(A)\right) \cap A$ and so $X \backslash U \subseteq c l_{w_{j}}(X \backslash A) \backslash(X \backslash A)$. Since $X \backslash A$ is $i j$-gwclosed, then by Theorem 8 , we have $X \backslash U=\emptyset$ and hence $U=X$.

Definition 4 If $c l_{w_{j}}\left(\cup_{\alpha} A_{\alpha}\right)=\cup_{\alpha} c l_{w_{j}}\left(A_{\alpha}\right)$, for $(j=1,2)$, then a family $\left\{A_{\alpha} \mid \alpha \in \Delta\right\}$ is called $w_{j}$-locally finite.

Theorem 13 Let $\left(X, w_{1}, w_{2}\right)$ be a biwss. If the family $\left\{A_{\alpha} \mid \alpha \in \Delta\right\}$ is $w_{j}$-locally finite, then the arbitrary union of $i j-g w c l o s e d ~ s e t s ~ A_{\alpha}, \alpha \in \Delta$ is an ij-gwclosed set.

Proof Direct to prove.

In the next definition, as an application of $j i$-gwopen sets, we offer a new type of sets namely $i j-\sigma g w$ closed sets.

Definition 5 A subset $A$ of a biwss $\left(X, w_{1}, w_{2}\right)$ is called ij-strongly generalized wclosed (briefly, ij-ogwclosed), if $c l_{w_{j}}(A) \subseteq U$, whenever $A \subseteq U$ and $U$ is ji-gwopen. The complement of $i j-\sigma$ gwclosed set is called $i j-\sigma g w o p e n$.

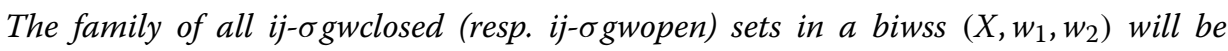
denoted by $i j-\sigma G W C(X)$ (resp. $i j-\sigma G W O(X)$ ).

Remark 8 If $A \in i j-\sigma G W C(X) \cap j i-\sigma G W C(X)$, then a subset $A$ of a biwss $\left(X, w_{1}, w_{2}\right)$ is called pairwise $\sigma$ gwclosed and its complement is called pairwise $\sigma$ gwopen.

For brevity the proof of the next proposition is omitted.

Proposition 5 In a biwss $\left(X, w_{1}, w_{2}\right)$, we have the following relation: $w_{j}$ closed set $\Rightarrow i j$ - $\sigma$ gwclosed set $\Rightarrow i j$-gwclosed set.

Remark 9 The converse of Proposition 5 is not true as can be seen from the next example.

Example 10 In Example 6, one may notice that $\{4\}$ is 21-gwclosed set, but it is not 21ogwclosed.

Example 11 In Example 8. One may notice that, $\{2\}$ is 12- $\sigma$ gwclosed set, but it is not $w_{2}$ closed.

Theorem 14 If $A \in j i-G W O(X) \cap i j-\sigma G W C(X)$, then $c l_{w_{j}}(A)=A$

Proof Straightforward.

Theorem 15 Let $c l_{w_{i}} \emptyset=\emptyset$. Then, $\{x\} \in j i-G W C(X)$ or $X \backslash\{x\} \in i j-\sigma G W C(X)$, for each $x \in X$. 
Proof Similar to Theorem 7.

Theorem 16 If $A \in i j-\sigma G W C(X)$, then $c l_{w_{j}}(A) \backslash A$ contains no nonempty ji-gwclosed.

Proof Similar to Theorem 8.

\section{Separation axioms in biweak spaces}

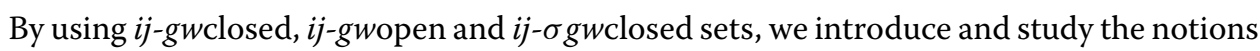
of $i j-w T_{\frac{1}{2}}, i j-w T_{\frac{1}{2}}^{\sigma}, i j-w^{\sigma} T_{\frac{1}{2}}, i j-w$ normal, and $i j-g w$ normal spaces.

Definition 6 Let $l_{w_{j}}(\emptyset)=\emptyset$. $A$ biwss $\left(X, w_{1}, w_{2}\right)$ is called

(1) $i j-w T_{1}$ if for each distinct points $x, y \in X$, there exist a $w_{i}$-open set $U$ and $w_{j}$-open set $V$ s.t. $x \in U, y \notin U$ and $y \in V, x \notin V$.

(2) ij-w $w T_{\frac{1}{2}}$ if each ij-gwclosed set $A$ of $X, c l_{w_{j}}(A)=A$.

(3) $i j-w T_{\frac{1}{2}}^{\sigma}$ if each ij- $\sigma$ gwclosed set $A$ of $X, c l_{w_{j}}(A)=A$.

Theorem 17 A biwss $\left(X, w_{1}, w_{2}\right)$ is ij-w $T_{1}$ if every singleton in $X$ is $i j$-wclosed.

Proof Let $x, y \in X$ and $x \neq y$. Then, $\{x\},\{y\}$ are $i j$-wclosed sets. From Theorem 1, we have $x \notin c l_{w_{i}}(\{y\})$ and $y \notin c l_{w_{j}}(\{x\})$. Hence, there exist $w_{i}$-open set $U$ containing $x$ and $w_{j}$-open set $V$ s.t. $x \in U, y \notin U$, and $y \in V, x \notin V$. Consequently, $\left(X, w_{1}, w_{2}\right)$ is a $i j-w T_{1}$ space.

In view of Proposition 5, the class of $i j-w T_{\frac{1}{2}}^{\sigma}$ spaces properly contains the class of $i j-w T_{\frac{1}{2}}$ spaces.

Proposition 6 Every $i j-w T_{\frac{1}{2}}$ space is $i j-w T_{\frac{1}{2}}^{\sigma}$.

The following example supports that the converse of the Proposition 6 is not true in general.

Example 12 In Example 5, $\left(X, w_{1}, w_{2}\right)$ is a $21-w T^{\sigma} \frac{1}{2}$ space but not $21-w T_{\frac{1}{2}}$.

Theorem 18 Let $X$ be a wiopen set and int $w_{j}\{x\}$ is $w_{j}$ open. A biwss $\left(X, w_{1}, w_{2}\right)$ is $i j-w T_{\frac{1}{2}}$ iff $\{x\}$ is $w_{i}$ closed or $\{x\}=$ int $_{w_{j}}\{x\}$ for each $x \in X$.

Proof Suppose that $\{x\}$ is not $w_{i}$ closed for some $x \in X$. Then, by using Theorem 7, $X \backslash\{x\}$ is $i j$-gwclosed. Since $\left(X, w_{1}, w_{2}\right)$ is $i j-w T_{\frac{1}{2}}$, then $\{x\}=i n t_{w_{j}}\{x\}$. On the other hand, let $B$ be an $i j-g w c l o s e d ~ s e t$. By assumption, $\{x\}$ is $w_{i}$ closed or $\{x\}=i n t_{w_{j}}\{x\}$ for any $x \in c l_{w_{j}} B$.

Case (I): Suppose $\{x\}$ is $w_{i}$ closed. If $x \notin B$, then $\{x\} \subseteq c l_{w_{j}} B \backslash B$, which is a contradiction to Theorem 8. Hence $x \in B$.

Case (II): Suppose $\{x\}=i n t_{w_{j}}\{x\}$ and $x \in \operatorname{cl}_{w_{j}} B$. Since $\{x\} \cap B \neq \emptyset$, we have $x \in B$. Thus, in both cases, we conclude that $c l_{w_{j}} B=B$. Therefore, $\left(X, w_{1}, w_{2}\right)$ is $i j-w T_{\frac{1}{2}}$ space.

Theorem 19 Suppose $c l_{w_{i}} \emptyset=\emptyset$. If $\left(X, w_{1}, w_{2}\right)$ is an ij-wT $T_{\frac{1}{2}}^{\sigma}$ space, then $\{x\}$ is ji-gwclosed or $\{x\}=\operatorname{int}_{w_{j}}\{x\}$, for each $x \in X$. 
Proof Follows directly from Theorem 15 and Definition 6.

Lemma 1 If $\{x\}$ is ji-gwclosed, then $\left(X, w_{1}, w_{2}\right)$ is an $i j-w-T_{\frac{1}{2}}^{\sigma}$ space, for each $x \in X$.

Proof Straightforward.

Definition 7 A biwss $\left(X, w_{1}, w_{2}\right)$ is called

(1) Pairwise $w T_{\frac{1}{2}}$ if it is both ij- $w T_{\frac{1}{2}}$ and ji-w $w T_{\frac{1}{2}}$.

(2) Pairwise $w T^{\frac{1}{2}} \frac{1}{2}$ if it is both ij-w $T_{\frac{1}{2}}^{\sigma}$ and ji- $w T_{\frac{1}{2}}^{\sigma}$.

Proposition 7 If $\left(X, w_{1}, w_{2}\right)$ is a pairwise $w T_{\frac{1}{2}}$ space, then it is pairwise $w T_{\frac{1}{2}}^{\sigma}$.

Proof Uncomplicated.

Remark 10 The converse of Proposition 7 is not true as can be seen from the next example.

Example 13 Let $X, w_{1}, w_{2}$ be as in Example 12. Then, $\left(X, w_{1}, w_{2}\right)$ is also a 21-w $T_{\frac{1}{2}}^{\sigma}$ space, and therefore, it is a pairwise $w T_{\frac{1}{2}}^{\sigma}$ space. But $\left(X, w_{1}, w_{2}\right)$ is not a pairwise $w T_{\frac{1}{2}}$ space.

Definition 8 A biwss $\left(X, w_{1}, w_{2}\right)$ is called an $i j-w^{\sigma} T_{\frac{1}{2}}$ if $i j-G W C(X)=i j-\sigma G W C(X)$.

Proposition 8 Every $i j-w T_{\frac{1}{2}}$ space is $i j-w^{\sigma} T_{\frac{1}{2}}$.

Proof Obvious.

Remark 11 The converse of Proposition 8 may not be applicable as we see in the next example.

Example 14 Let $X=\{1,2,3,4\}$. Define weak structures $w_{1}, w_{2}$ on $X$ as follows: $w_{1}=\{\emptyset,\{1,3\},\{1,4\},\{2,3,4\}\}$ and $w_{2}=\{\emptyset,\{2\},\{1,2\},\{3,4\},\{1,3,4\}\}$. Then, $\left(X, w_{1}, w_{2}\right)$ is an $12-w^{\sigma} T_{\frac{1}{2}}$ space but not $12-w T_{\frac{1}{2}}$.

Remark $12 i j-w^{\sigma} T_{\frac{1}{2}}$ and $i j-w T_{\frac{1}{2}}^{\sigma}$ spaces are independent as may be seen from Example 15 and Example 16.

Example 15 Let $w_{1}=\{\emptyset,\{1\},\{1,2\}\}, w_{2}=\{\emptyset,\{3\}, X\}$ be weak structures on $X=\{1,2,3\}$, then $\left(X, w_{1}, w_{2}\right)$ is a $12-w T^{\sigma} \frac{1}{2}$ space but not $12-w^{\sigma} T_{\frac{1}{2}}$.

Example 16 In Example 14, $\left(X, w_{1}, w_{2}\right)$ is an $12-w^{\sigma} T_{\frac{1}{2}}$, but it is not $12-w-T_{\frac{1}{2}}^{\sigma}$.

Theorem 20 Let $l_{w_{j}}(\emptyset)=\emptyset$. A biwss $\left(X, w_{1}, w_{2}\right)$ is $i j-w T_{\frac{1}{2}}$ if and only if it is both $i j-w T_{\frac{1}{2}}^{\sigma}$ and $i j-w^{\sigma} T_{\frac{1}{2}}$ space.

Proof Suppose that $\left(X, w_{1}, w_{2}\right)$ is an $i j-w T_{\frac{1}{2}}$ space. Then, by Propositions 6 and 8, $\left(X, w_{1}, w_{2}\right)$ is both $i j-w T_{\frac{1}{2}}^{\sigma}$ and $i j-w^{\sigma} T_{\frac{1}{2}}$ space. Conversely, suppose that $\left(X, w_{1}, w_{2}\right)$ is both 
$i j-w T_{\frac{1}{2}}^{\sigma}$ and $i j-w^{\sigma} T_{\frac{1}{2}}$. Let $A \in i j-G W C(X)$. Since $\left(X, w_{1}, w_{2}\right)$ is an $i j-w^{\sigma} T_{\frac{1}{2}}$ space, $A \in i j-$ $\sigma G W C(X)$. Since $\left(X, w_{1}, w_{2}\right)$ is an $i j-w T_{\frac{1}{2}}^{\sigma}$ space, then $c l_{w_{j}}(A)=A$. Therefore, $\left(X, w_{1}, w_{2}\right)$ is $i j-w T_{\frac{1}{2}}$.

Definition $9 A$ biwss $\left(X, w_{1}, w_{2}\right)$ is called ij-wnormal if for each $w_{i}$ closed set $A$ and $w_{j}$ closed set $B$ s.t. $A \cap B=\emptyset$, there are $w_{j}$ open set $U$ and $w_{i}$ open set $V$ s.t. $A \subseteq U, B \subseteq V$, and $U \cap V=\emptyset$.

Theorem 21 Let $\left(X, w_{1}, w_{2}\right)$ be a biwss. Consider the following statements:

(1) $\left(X, w_{1}, w_{2}\right)$ is ij-wnormal,

(2) For each $w_{i}$ closed set $A$ and $w_{j}$ open set $N$ with $A \subseteq N$, there exists $w_{j}$ open set $U$ s.t. $A \subseteq U \subseteq c l_{w_{i}}(U) \subseteq N$,

(3) For each $w_{i}$ closed set $A$ and each ij-gwclosed set $H$ with $A \cap H=\emptyset$, there exist $w_{j}$ open set $U$ and $w_{i}$ open set $V$ s.t. $A \subseteq U, H \subseteq V$ and $U \cap V=\emptyset$,

(4) For each $w_{i}$ closed set $A$ and ij-gwopen $N$ with $A \subseteq N$, there exists $w_{j}$ open set $U$ s.t. $A \subseteq U \subseteq c l_{w_{i}}(U) \subseteq N$.

Then, the implications (1) $\Rightarrow$ (2) and (3) $\Rightarrow(4) \Rightarrow$ (2) are hold.

Proof Obvious.

Theorem 22 Let $\left(X, w_{1}, w_{2}\right)$ be a biwss. If $c_{w_{i}}(A)$ is $w_{i}$ closed for each $w_{j}$ open or ij-gwclosed, then the statements in Theorem 21 are equivalent.

Proof According to Theorem 21, we need to prove (2) $\Rightarrow$ (1) and (1) $\Rightarrow$ (3) only. $(2) \Rightarrow(1)$ : Let $A$ be a $w_{i}$ closed set and $B$ be a $w_{j}$ closed set with $A \cap B=\emptyset$. Then, $X \backslash B$ is a $w_{j}$ open set with $A \subseteq X \backslash B$. Thus, by (2) there exists $w_{j}$ open set $U$ s.t. $A \subseteq U \subseteq c l_{w_{i}}(U) \subseteq X \backslash B$. Hence $A \subseteq U$ and $B \subseteq X \backslash c l_{w_{i}}(U)$. Since $c l_{w_{i}}(U)$ is $w_{i}$ closed for each $w_{j}$ open $U$, then $X \backslash c l_{w_{i}}(U)=V$ is $w_{i}$ open and $U \cap V=\emptyset$. Hence $\left(X, w_{1}, w_{2}\right)$ is $i j$-wnormal.

(1) $\Rightarrow$ (3): Let $A$ be a $w_{i}$ closed set and $H$ be an $i j-g w c l o s e d ~ s e t$ with $A \cap H=\emptyset$. Then, $H \subseteq X \backslash A$. From Definition 3, we have $c l_{w_{j}}(H) \subseteq X \backslash A$. Since $H$ is $i j$-gwclosed, then $c l_{w_{j}}(H)$ is $w_{j}$ closed. Since $A \cap c l_{w_{j}}(H)=\emptyset$, then from (1) there exist $w_{j}$ open set $U$ and $w_{i}$ open set $V$ s.t. $A \subseteq U, H \subseteq c l_{w_{j}}(H) \subseteq V$ and $U \cap V=\emptyset$.

Theorem 23 Let $\left(X, w_{1}, w_{2}\right)$ be a biwss. Consider the following statements:

(1) $\left(X, w_{1}, w_{2}\right)$ is ij-wnormal,

(2) For each $w_{i}$ closed set $A$ and $w_{j}$ closed set $B$ s.t. $A \cap B=\emptyset$, there exist ij-gwopen $U$ and ji-gwopen $V$ s.t. $A \subseteq U, B \subseteq V$ and $U \cap V=\emptyset$,

(3) For each $w_{i}$ closed set $A$ and $w_{j}$ open $N$ with $A \subseteq N$, there exists ij-gwopen $U$ s.t. $A \subseteq U \subseteq c l_{w_{i}}(U) \subseteq N$.

Then, the implication (1) $\Rightarrow(2) \Rightarrow$ (3) is hold.

Proof $(1) \Rightarrow$ (2): Let $A$ be a $w_{i}$ closed set and $B$ be a $w_{j}$ closed set with $A \cap B=\emptyset$. Since $\left(X, w_{1}, w_{2}\right)$ is $i j$-wnormal, then there exist $w_{j}$ open set $U$ and $w_{i}$ open set $V$ s.t. $A \subseteq U, B \subseteq V$ and $U \cap V=\emptyset$. From Corollary 1 , there exist $i j$-gwopen $U$ and ji-gwopen $V$ s.t. $A \subseteq U, B \subseteq V$ and $U \cap V=\emptyset$. 
(2) $\Rightarrow$ (3): Let $A$ be a $w_{i}$ closed set and $N$ be a $w_{j}$ open set with $A \subseteq N$. Then, $A \cap X \backslash N=\emptyset$. From (2), there exist $i j$-gwopen $U$ and ji-gwopen $V$ s.t. $A \subseteq U, X \backslash N \subseteq V$, and $U \cap V=\emptyset$. Since $X \backslash V$ is ji-gwclosed, $N$ is $w_{j}$ open, and $X \backslash V \subseteq N$, then from Definition 3, we have $c l_{w_{i}}(X \backslash V) \subseteq N$. Since $U \subseteq X \backslash V$, hence $U \subseteq c l_{w_{i}}(U) \subseteq c l_{w_{i}}(X \backslash V)$. Consequently, $A \subseteq U \subseteq c l_{w_{i}}(U) \subseteq N$.

Theorem 24 Let $\left(X, w_{1}, w_{2}\right)$ be an $i j-w T_{\frac{1}{2}}$. If $c l_{w_{i}}(U)$ is $w_{i}$ closed for each ij-gwclosed and int $_{w_{j}}(U)$ is $w_{j}$ open for each ij-gwclosed $U$, then the statements in Theorem 23 are equivalent.

Proof According to Theorem 23, we need to prove (3) $\Rightarrow$ (1).

(3) $\Rightarrow$ (1): Let $A$ be a $w_{i}$ closed set and $B$ be a $w_{j}$ closed set with $A \cap B=\emptyset$. Take $N=X \backslash B$, then by using (3) there exists $i j$-gwopen $U$ s.t. $A \subseteq U \subseteq c l_{w_{i}}(U) \subseteq N$. Since $\left(X, w_{1}, w_{2}\right)$ is an $i j-w T_{\frac{1}{2}}$ space, then, $i n t_{w_{j}}(U)=U$. By assumption $U$ is $w_{j}$ open. Also, $X \backslash c l_{w_{i}}(U)$ is $w_{i}$ open and $B \subseteq X \backslash c l_{w_{i}}(U)$.

Definition $10 A$ biwss $\left(X, w_{1}, w_{2}\right)$ is called ij-gwnormal iffor each $j i$-gwclosed set $A$ and ij-gwclosed set $B$ s.t. $A \cap B=\emptyset$, there are $w_{j}$ open set $U$ and $w_{i}$ open set $V$ s.t. $A \subseteq U, B \subseteq V$ and $U \cap V=\emptyset$.

Remark 13 It is clear that every ij-gwnormal space is ij-wnormal. It can be checked that the converse is not true by the following example.

Theorem 25 Let $\left(X, w_{1}, w_{2}\right)$ be a biwss. Consider the following statements:

(1) $\left(X, w_{1}, w_{2}\right)$ is ij-gwnormal,

(2) For each ji-gwclosed set $A$ and ij-gwopen set $N$ with $A \subseteq N$, there exists $w_{j}$ open set $U$ s.t. $A \subseteq U \subseteq c l_{w_{i}}(U) \subseteq N$,

(3) For each ji-gwclosed set $A$ and ij-gwclosed set $B$ s.t. $A \cap B=\emptyset$, there exist $w_{j}$ open set $U$ s.t. $A \subseteq U$ and $c l_{w_{i}}(U) \cap B=\emptyset$.

Then, the implication (1) $\Rightarrow(2) \Rightarrow$ (3) is hold.

Proof Obvious.

Remark 14 If $c l_{w_{i}}(U)$ is $w_{i}$ closed for each $w_{i}$ open set $U$, then the statements in Theorem 25 are equivalent.

Theorem 26 Let $\left(X, w_{1}, w_{2}\right)$ be a biwss. Consider the following statements:

(1) $\left(X, w_{1}, w_{2}\right)$ is ij-gwnormal,

(2) For each ji-gwclosed set $A$ and ij-gwclosed set $B$ s.t. $A \cap B=\emptyset$, there exist ij- $\sigma$ gwopen set $U$, ji- $\sigma$ gwopen set $V$ s.t. $A \subseteq U, B \subseteq V$ and $U \cap V=\emptyset$,

(3) For each ji-gwclosed set $A$ and ij-gwopen set $N$ with $A \subseteq N$, there exists ij- $\sigma$ gwopen set $U$ s.t. $A \subseteq U \subseteq c l_{w_{i}}(U) \subseteq N$.

Then, the implication (1) $\Rightarrow(2) \Rightarrow$ (3) is hold. 
Proof $(1) \Rightarrow$ (2) Follows directly from Proposition 5.

(2) $\Rightarrow$ (3) Let $A$ be a ji-gwclosed set and $N$ be an $i j$-gwopen set with $A \subseteq N$. Take $B=X \backslash N$. Then, by assumption, there exist $i j-\sigma g w o p e n$ set $U$, ji- $\sigma g w o p e n$ set $V$ s.t. $A \subseteq U, B \subseteq V$ and $U \cap V=\emptyset$. Hence, $U \subseteq X \backslash V, X \backslash V \subseteq N$. Since $X \backslash V$ is $j i$ - $\sigma g w c l o s e d$, then $c_{w_{i}}(X \backslash V) \subseteq N$ and so $A \subseteq U \subseteq c l_{w_{i}}(U) \subseteq N$.

The question that comes to our mind, under what conditions can be achieved parity in Theorem 26.

Theorem 27 Let $\left(X, w_{1}, w_{2}\right)$ be an ij-w-T $T_{\frac{1}{2}}^{\sigma}$ space. If int $_{w_{j}}(U)$ is $w_{j}$ open and int $_{w_{i}}(U)$ is $w_{i}$ open for each ij- $\sigma$ gwopen set $U$, then the statements in Theorem 26 are equivalent.

Proof Straightforward.

Corollary 4 If a biwss $\left(X, w_{1}, w_{2}\right)$ is ij-gwnormal, then for each ji-gwclosed set $A$ and $i j-\sigma g$ wopen set $N$ with $A \subseteq N$, there exists ij- $\sigma$ gwopen set $U$ s.t. $A \subseteq U \subseteq c l_{w_{i}}(U) \subseteq N$.

Proof Obvious from Proposition 5.

Theorem 28 If a biwss $\left(X, w_{1}, w_{2}\right)$ is ij-gwnormal, then for each ji-gwclosed set $A$ and $i j-$ gwclosed set $B$ s.t. $A \cap B=\emptyset$, there exist ij-gwopen set $U$ and ji-gwopen set $V$ s.t. $A \subseteq U, B \subseteq V$ and $U \cap V=\emptyset$.

Proof Clear.

Theorem 29 If a biwss $\left(X, w_{1}, w_{2}\right)$ is ji-w-T $T_{\frac{1}{2}}^{\sigma}$ and $c l_{w_{i}}(\emptyset)=\emptyset$. Consider the following statements:

(1) $\left(X, w_{1}, w_{2}\right)$ is ij-gwnormal,

(2) For each ji-gwclosed set $A$ and ij-gwopen set $N$ with $A \subseteq N$, there exists ij-gwopen set $U$ s.t. $A \subseteq U \subseteq c l_{w_{i}}(U) \subseteq N$.

Then, the implication (1) $\Rightarrow$ (2) is hold.

Proof Obvious.

\section{Some types of $i j-\left(w, w^{\star}\right)$ continuous functions}

In this section, types of continuous functions between biweak spaces are defined and some of their features are established.

Definition 11 A function $f:\left(X, w_{1}, w_{2}\right) \longrightarrow\left(Y, w_{1}^{\star}, w_{2}^{\star}\right)$ is called:

(1) $j$ - $\left(w, w^{\star}\right)$-continuous if for $x \in X$ and $w_{j}^{\star}$ open set $V$ containing $f(x)$, there is a $w_{j}$ open set $U$ containing $X$ s.t. $f(U) \subseteq V$.

(2) $\operatorname{ij}-g\left(w, w^{\star}\right)$-continuous if for $x \in X$ and $w_{j}^{\star}$ open set $V$ containing $f(x)$, there is an ij-gwopen set $U$ containing $x$ s.t. $f(U) \subseteq V$.

(3) $i j-g\left(w, w^{\star}\right)$ closed if for each $w_{j}$ closed set $B, f(B)$ is ji-g $w^{\star}$ closed set.

We describe $i j-g\left(w, w^{\star}\right)$-continuous function in the following part. 
Theorem 30 Let $\left(X, w_{1}, w_{2}\right)$ be an $i j-w T_{\frac{1}{2}}$ space. If int $_{w_{j}}(U)$ is $w_{j}$ open for each $i j-$ gwopen set $U$, then, a function $f:\left(X, w_{1}, w_{2}\right) \longrightarrow\left(Y, w_{1}^{\star}, w_{2}^{\star}\right)$ is ij-g( $\left(w, w^{\star}\right)$-continuous iff $f^{-1}(V)=\operatorname{int}_{w_{j}} f^{-1}(V)$ for each $w_{j}^{\star}$ open set $V$.

Proof $(\Rightarrow)$ : Let $V$ be a $w_{j}^{\star}$ open set and $x \in f^{-1}(V)$. Since $f$ is $i j-g\left(w, w^{\star}\right)$-continuous, then there is an $i j$-gwopen set $U$ containing $x$ s.t. $f(U) \subseteq V$. Hence, $U \subseteq f^{-1}(V)$. Since $\left(X, w_{1}, w_{2}\right)$ is an $i j-w T_{\frac{1}{2}}$ space, then $i n t_{w_{j}}(U)=U$. From assumptions, $U$ is a $w_{j}$ open set s.t. $x \in U \subseteq f^{-1}(V)$ and so $x \in$ int $_{w_{j}} f^{-1}(V)$. Therefore, $f^{-1}(V)=i n t_{w_{j}} f^{-1}(V)$.

$(\Leftarrow)$ : Let $x \in X$ and $V$ be a $w_{j}^{\star}$ open set in $Y$ with $f(x) \in V$, then $x \in f^{-1}(V)$. Since $f^{-1}(V)=\operatorname{int}_{w_{j}} f^{-1}(V)$, then there exists $w_{j}$ open set $U$ s.t. $x \in U \subseteq f^{-1}(V)$. From Corollary $1, U$ is an $i j-g w o p e n$ set containing $x$ s.t. $f(U) \subseteq V$. Consequently, $f$ is $i j-g\left(w, w^{\star}\right)$-continuous.

Theorem 31 For a function $f:\left(X, w_{1}, w_{2}\right) \longrightarrow\left(Y, w_{1}^{\star}\right.$, $\left.w_{2}^{\star}\right)$, the following are equivalent:

(1) $f^{-1}(V)=\operatorname{int}_{w_{j}}\left(f^{-1}(V)\right)$, for every $w_{j}^{\star}$ open set $V$ in $Y$,

(2) $f\left(c l_{w_{j}}(A)\right) \subseteq c l_{w_{j}^{\star}}(f(A))$, for every set $A$ in $X$,

(3) $c l_{w_{j}}\left(f^{-1}(V)\right) \subseteq\left(f^{-1}\left(c l_{w_{j}^{*}}(V)\right)\right.$, for every set $V$ in $Y$,

(4) $f^{-1}\left(\operatorname{int}_{w_{j}^{*}}(V)\right) \subseteq \operatorname{int}_{w_{j}}\left(f^{-1}(V)\right)$, for every set $V$ in $Y$,

(5) $c l_{w_{j}}\left(f^{-1}(F)\right)=f^{-1}(F)$, for every $w_{j}^{\star}$ closed set $F$ in $Y$.

Proof Obvious.

Theorem 32 For any function $f:\left(X, w_{1}, w_{2}\right) \longrightarrow\left(Y, w_{1}^{\star}, w_{2}^{\star}\right)$, every $j-\left(w, w^{\star}\right)$-continuous function is $i j-g\left(w, w^{\star}\right)$-continuous.

Proof Obvious from Theorem 30.

Remark 15 The following example justifies the converse of the Theorem 32 need not to be true in general.

Example 17 Let $X=\{a, b, c, d\}, \quad Y=\{1,2,3\}, \quad w_{1}=\{\emptyset,\{a\},\{a, d\}\}, \quad w_{2}=\{\emptyset,\{a, b\},\{c, d\}\}$, $w_{1}^{\star}=\{\emptyset,\{1\},\{2,3\}\}$, and $w_{2}^{\star}=\{\emptyset,\{2\},\{1,2\}\}$. If is defined by $f(a)=f(b)=2, f(c)=1, f(d)=3$, we have $f$ is $12-g\left(w, w^{\star}\right)$-continuous, but it is not $2-\left(w, w^{\star}\right)$-continuous.

Proposition 9 For any surjection function $f:\left(X, w_{1}, w_{2}\right) \longrightarrow\left(Y, w_{1}^{\star}, w_{2}^{\star}\right)$, the following are equivalent.

(1) $f$ is an ij- $g\left(w, w^{\star}\right)$ closed function.

(2) For any set $B$ in $Y$ and each $w_{i}$ open $U$ s.t. $f^{-1}(B) \subseteq U$, there exists ij-gw $w^{\star}$ open set $V$ of $Y$ s.t. $B \subseteq V$ and $f^{-1}(V) \subseteq U$.

Proof (1) $\Rightarrow$ (2): Let $B \subseteq Y$ and $U$ be a $w_{i}$ open set s.t. $f^{-1}(B) \subseteq U$. Since $f$ is an $i j$ $g\left(w, w^{\star}\right)$ closed function, then $f(U)$ is an $i j-g w^{\star}$ open set in $Y$. Take $f^{-1}(V)=U$. Since $f$ is a surjection function and $f^{-1}(B) \subseteq U$, then $B=f\left(f^{-1}(B)\right) \subseteq f(U)=V$.

$(2) \Rightarrow(1)$ : Let $U$ be a $w_{i}$ open set, $F \subseteq f(U)$ s.t. $F$ is a $w_{i}^{\star}$ closed set, then $f^{-1}(F) \subseteq U$. This implies that there exists $i j-g w^{\star}$ open set $V$ in $Y$ s.t. $F \subseteq V$ and $f^{-1}(V) \subseteq U$. Consequently, 
$F \subseteq \operatorname{int}_{w_{j}^{\star}}(V)$ and so $F \subseteq i n t_{w_{j}^{\star}}(f(U))$. This implies that $f(U)$ is $i j-g w^{\star}$ open in $Y$. Therefore, $f$ is an $i j-g\left(w, w^{\star}\right)$ closed function.

Theorem 33 Let $\left(Y, w_{1}^{\star}, w_{2}^{\star}\right)$ be an ij- $w^{\star} T_{\frac{1}{2}}$ space. If int $_{w_{j}^{\star}}(A)$ is $w_{i}^{\star}$ open for each $i j$ gw $w^{\star}$ open set A. If $f:\left(X, w_{1}, w_{2}\right) \longrightarrow\left(Y, w_{1}^{\star}, w_{2}^{\star}\right)$ is a surjection ij-g $\left(w, w^{\star}\right)$ closed and $i j-g\left(w, w^{\star}\right)$-continuous function, then $f^{-1}(B)$ is ij-gwclosed set of $X$ for every ij-g $g w^{\star}$ closed set $B$ of $Y$.

Proof Let $B \subseteq Y$ be an $i j-g w^{\star}$ closed set. Let $U$ be a $w_{i}$ open set of $X$ s.t. $f^{-1}(B) \subseteq U$. Since $f$ is a surjection $i j-g\left(w, w^{\star}\right)$ closed function, then by Proposition 9 , there exists $i j$ $g w^{\star}$ open set $V$ of $Y$ s.t. $B \subseteq V$ and $f^{-1}(V) \subseteq U$. Since $\left(Y, w_{1}^{\star}\right.$, $\left.w_{2}^{\star}\right)$ is an $i j-w^{\star} T_{\frac{1}{2}}$ space, then int $_{w_{j}^{\star}}(V)=V$. From assumptions, $V$ is a $w_{i}^{\star}$ open set. Since $B$ is $i j-g w^{\star}$ closed, then $c l_{w_{j}^{\star}}(B) \subseteq$ $V$. Hence, $f^{-1}\left(c l_{w_{j}^{*}}(B)\right) \subseteq f^{-1}(V) \subseteq U$. By Theorems 30 and 31, $c l_{w_{j}} f^{-1}(B) \subseteq U$, and hence, $f^{-1}(B)$ is $i j-g w c l o s e d$ set in $X$.

Lemma 2 Let $\left(Y, w_{1}^{\star}, w_{2}^{\star}\right)$ be an $j i-w^{\star} T_{\frac{1}{2}}$ space. If $:\left(X, w_{1}, w_{2}\right) \longrightarrow\left(Y, w_{1}^{\star}, w_{2}^{\star}\right)$ is an $i j-g\left(w, w^{\star}\right)$ closed function, then $c l_{w_{i}^{\star}} f(A)=f\left(c l_{w_{j}}(A)\right)$, for every $w_{j}$ closed set $A$ in $X$.

Proof Let $A$ be a $w_{j}$ closed set in $X$, then $A=c l_{w_{j}}(A)$. Since $f$ is an $i j-g\left(w, w^{\star}\right)$ closed function, then $f(A)$ is $j i-g w^{\star}$ closed set since $\left(Y, w_{1}^{\star}, w_{2}^{\star}\right)$ is a $j i-w^{\star} T_{\frac{1}{2}}$ space, then $c l_{w_{i}^{\star}} f(A)=f(A)$. Hence, $c l_{w_{i}^{\star}} f(A)=f\left(c l_{w_{j}}(A)\right)$.

Lemma 3 Let $\left(Y, w_{1}^{\star}, w_{2}^{\star}\right)$ be an $i j-w^{\star} T_{\frac{1}{2}}$ space. and $f:\left(X, w_{1}, w_{2}\right) \longrightarrow\left(Y, w_{1}^{\star}, w_{2}^{\star}\right)$ be a ji-g $\left(w, w^{\star}\right)$ closed function. If $c l_{w_{j}}(A)$ is a $w_{i}$ closed set for each set $A$ in $X$, then $c l_{w_{j}^{\star}} f(A) \subseteq$ $f\left(c l_{w_{j}}(A)\right)$.

Proof Suppose $c l_{w_{j}}(A)$ is a $w_{i}$ closed set in $X$. Since $f$ is an $j i-g\left(w, w^{\star}\right)$ closed function, then $f\left(c l_{w_{j}}(A)\right)$ is $i j-g w^{\star}$ closed set containing $f(A)$. Since $\left(Y, w_{1}^{\star}, w_{2}^{\star}\right)$ is an $i j-w^{\star} T_{\frac{1}{2}}$ space, then $c l_{w_{j}^{\star}} f\left(c l_{w_{j}}(A)\right)=f\left(c l_{w_{j}}(A)\right)$. Hence, $c l_{w_{j}^{\star}} f(A) \subseteq f\left(c l_{w_{j}}(A)\right)$.

Theorem 34 Let $\left(Y, w_{1}^{\star}, w_{2}^{\star}\right)$ be an ij- $w^{\star} T_{\frac{1}{2}}$ space. If int $w_{w_{i}} f^{-1}(U)$ is $w_{i}$ open for each $w_{i}^{\star}$ open set $U$ in $Y$ and $c l_{w_{j}}(A)$ is a $w_{i}$ closed set for each set $A$ in $X$. Iff $:\left(X, w_{1}, w_{2}\right) \longrightarrow$ $\left(Y, w_{1}^{\star}, w_{2}^{\star}\right)$ is a ji-g $\left(w, w^{\star}\right)$ closed and ji-g $\left(w, w^{\star}\right)$-continuous function, then $f(A)$ is ijg $w^{\star}$ closed set of $Y$ for every ij-gwclosed set $A$ of $X$.

Proof Follows directly from Theorem 30, Theorem 31, and Lemma 3.

Theorem 35 Let $\left(Y, w_{1}^{\star}\right.$, $\left.w_{2}^{\star}\right)$ be an $i j-w^{\star} T_{\frac{1}{2}}$ space. If $i_{w_{j}^{\star}}(A)$ is $w_{j}^{\star}$ open for each $i j-g w^{\star}$ open set $A$ of $Y$. If $f:\left(X, w_{1}, w_{2}\right) \longrightarrow\left(Y, w_{1}^{\star}, w_{2}^{\star}\right)$ and $h:\left(Y, w_{1}^{\star}, w_{2}^{\star}\right) \longrightarrow\left(Z, v_{1}, v_{2}\right)$ are $i j-g\left(w, w^{\star}\right)$-continuous and $i j-g\left(w^{\star}, v\right)$-continuous functions, respectively, then $h \circ f$ : $\left(X, w_{1}, w_{2}\right) \longrightarrow\left(Z, v_{1}, v_{2}\right)$ is ij-g(w,v)-continuous.

Proof Let $x \in X$ and $V$ be a $v_{j}$ open set of $Z$ containing $h \circ f(x)$. Since $h$ is $i j-g\left(w^{\star}, v\right)$ continuous, then there is an $i j-g w^{\star}$ open set $U$ containing $h(x)$ s.t. $h(U) \subseteq V$. Since $\left(Y, w_{1}^{\star}, w_{2}^{\star}\right)$ is an $i j-w^{\star} T_{\frac{1}{2}}$ space, hence, $i_{w_{j}^{\star}}(U)=U$. From assumptions, $U$ is a $w_{j}^{\star}$ open for each $i j-g w^{\star}$ open set $U$ of $Y$ containing $h(x)$. Since $f$ is an $i j-g\left(w, w^{\star}\right)$-continuous function, 
so there is an $i j$-gwopen set $G$ containing $x$ s.t. $f(G) \subseteq U$. It follows that there exists an $i j$ $g w o p e n$ set $G$ containing $x$ s.t. $h \circ f(G) \subseteq V$. Consequently, $h \circ f$ is $i j-g(w, v)$-continuous.

Theorem 36 If $f:\left(X, w_{1}, w_{2}\right) \longrightarrow\left(Y, w_{1}^{\star}, w_{2}^{\star}\right)$ and $h:\left(Y, w_{1}^{\star}, w_{2}^{\star}\right) \longrightarrow\left(Z, v_{1}, v_{2}\right)$ are $i j-g\left(w, w^{\star}\right)$-continuous and $j-\left(w^{\star}, v\right)$-continuous respectively, then hof $:\left(X, w_{1}, w_{2}\right) \longrightarrow$ $\left(Z, v_{1}, v_{2}\right)$ is ij-g(w,v)-continuous.

Proof Straightforward.

\section{Future work}

In the future, we intend to introduce the bisoft weak structure spaces and study the

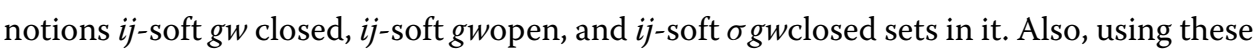
sets, diverse classes of mappings on soft biweak structures can be examined. Further, we suggest studying the properties of some kinds of $i j$-gwclosed subsets with respect to a biweak structure modified by elements of an ideal or a hereditary class. Accordingly, we construct a kind of continuity depending on the new class of $i j$ - $g w$ closed subsets. Moreover, one may take research to find the suitable way of defining the biweak structure spaces associated to the digraphs by using $i j$-gwclosed such that there is a one-to-one correspondence between them. It may also lead to the new properties of separation axioms on these spaces. It will be necessary to perform more research to strengthen a comprehensive framework for the practical applications.

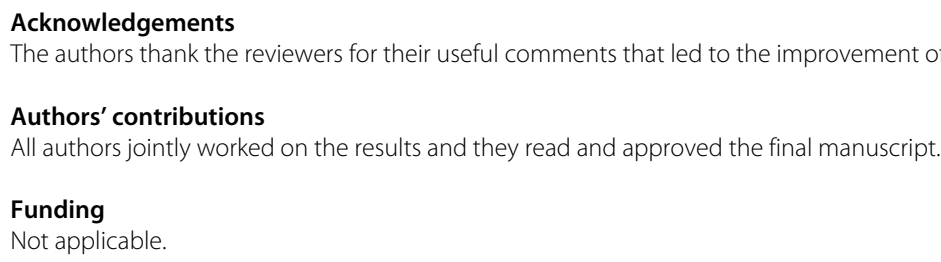

11. Al-Omari, A, Noiri, T: weak continuity between WSS and GTS due to Csaszar. Malays. J. Math. Sci. 7(2), 297-313 (2013)

\section{Publisher's Note}

Springer Nature remains neutral with regard to jurisdictional claims in published maps and institutional affiliations. 